\title{
Transformarse en médico: narrativas de discontinuidad $y$ argumentos autobiográficos
}

Transforming into a physician: discontinuity narratives and autobiographical arguments

Marcia Villanueva Lozano

(UNAM, CRIM) marcia.villanueva.lozano@gmail.com

DOI: https://doi.org/10.15366/bp2021.28.009

Bajo Palabra. II Época. No28. Pgs: 199-216 Orcid: https://orcid.org/0000-0001-9408-6308 
Recibido: 30/09/2021

Aceptado: 12/11/2021

Este artículo ha sido posible gracias al Proyecto de Investigación Institución y

Constitución de la Individualidad: Aspectos Ontológicos, Sociales y de Derecho (PID2020-117413GA-I00 / AEI / 10.13039 / 501100011033).

Investigadora postdoctoral, UNAM. Programa de Becas Posdoctorales en la

UNAM, Becaria del Centro Regional de Investigaciones Multidisciplinarias

(CRIM), asesorada por el Dr. Roberto Castro.

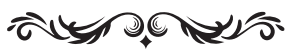

Resumen

Este artículo presenta una aproximación al problema de la persistencia desde un enfoque narrativo de la identidad personal para caracterizar lo que denomino el problema de la transformación. Analizo este problema utilizando experiencias de médicos y estudiantes de medicina mexicanos que describen cómo fue para ellos "transformarse en médico". Con estos datos empíricos, exploro las narrativas de discontinuidad que surgen con la transformación, y los argumentos autobiográficos que se arguyen para reparar la discontinuidad.

Palabras clave: Identidad narrativa, problema de la persistencia, transformación, discontinuidad, argumentos autobiográficos.

\section{Abstract}

This paper presents an account on the persistence problem from a narrative perspective to describe what I call the transformation problem. The analysis is based on medical students' and professionals' experiences regarding how it was for them "transforming into a physician". By using these empirical data, this paper explores the discontinuity narratives and the autobiographical arguments used to repair such discontinuity.
Keywords: Narrative identity, persistence problem, transformation, discontinuity, autobiographical arguments. 


\section{Introducción}

El problema de la persistencia de la identidad personal se refiere a la cuestión de la individualidad a lo largo del tiempo, por lo que también se conoce como el problema de la identidad diacrónica. La pregunta de la que se encarga es ¿cuándo una persona sobrevive o muere?, cuyo reto fundamental consiste en establecer no los límites de la vida biológica, sino cuándo una persona sigue siendo la misma persona (Olson, 2010).

Dicho problema ha ocupado a muchos filósofos desde que Locke planteó el experimento mental del príncipe y el zapatero. Este formula un intercambio de conciencia entre estos dos personajes, de tal suerte que la conciencia del príncipe entra al cuerpo del zapatero y viceversa, para enseguida preguntar ¿quién es el príncipe: aquel que tiene su cuerpo o aquel otro que tiene su conciencia? Locke llega a la conclusión de que este último es el príncipe en virtud de que "todo lo que tenga la conciencia de acciones presentes y pasadas es la misma persona" (Locke 1982[1690]:324; énfasis mío).

Desde entonces a la fecha, muchos otros filósofos han planteado diversos y creativos experimentos mentales que incluyen fisiones, duplicaciones, teletransportaciones, trasplantes cerebrales, entre otros, para establecer en qué casos sobrevive (o no) una persona. ${ }^{1}$ Este tipo de abordajes constituye el planteamiento clásico del problema de la persistencia, el cual busca dar respuesta a lo que Schechtman (1996) llama la pregunta de la reidentificación: la pregunta metafísica de cómo un individuo persiste a través del tiempo, es decir, aquella que indaga las condiciones necesarias y suficientes para que una persona en un tiempo T1 sea la misma persona en el tiempo T2. ${ }^{2}$

El problema de la persistencia lleva implícita otra pregunta que ha recibido mucha menos atención, denominada por Schechtman (1996) la pregunta de la caracterización. Esta indaga qué acciones, experiencias, creencias, valores, rasgos de carácter y otros elementos constituyen la identidad de una persona. En este artículo argumentaré que, desde esta perspectiva, el problema de la persistencia ya no se plantea como una cuestión de sobrevivencia, sino como un problema de

${ }^{1}$ Ver Wilkes (1993) para una discusión sobre el uso de experimentos mentales en la filosofía de la identidad personal y Dennett (1978) para una sátira de estos experimentos mentales.

2 Este tema ha sido tratado con profusión por Muñoz Corcuera, véase, por ejemplo, Muñoz Corcuera (2021). 
transformación debido a cambios en la caracterización de una persona. Desafiando la metodología tradicional de la filosofía de la identidad personal asentada en experimentos mentales, exploraré el problema de la transformación con base en datos empíricos de mi investigación sobre la deshumanización de la medicina y la formación profesional de los médicos (Villanueva, 2019). Desde un enfoque narrativo de la identidad personal (Lindemann, 2001 y 2014), utilizaré experiencias de médicos y estudiantes de medicina mexicanos ${ }^{3}$ que describen cómo fue para ellos "transformarse en médico" para analizar sus narrativas de discontinuidad y los cambios implicados en su identidad. Por último, hablaré sobre la resistencia al cambio y los argumentos autobiográficos que sirven para reparar la discontinuidad.

\section{El problema de la transformación}

Schechtman (1996) argumenta que algunos de los experimentos mentales expuestos por los filósofos para responder a la pregunta de la reidentificación son más compatibles con la pregunta de la caracterización. Como ejemplo toma el caso del joven ruso del siglo XIX planteado por Parfit (1984). En este escenario imaginario, un noble joven ruso, que heredará mucha tierra y tiene gran simpatía por los campesinos, teme en un futuro ser corrompido por la riqueza, por lo que redacta un documento legal con el que cede a los campesinos las tierras que heredará. Este documento sólo podrá ser revocado por su esposa, a quien le solicita que no disuelva la concesión de las tierras, sin importar cuánto él le ruegue, pues explica: "yo considero que mis ideales me son esenciales. Si pierdo estos ideales, quiero que pienses que yo dejé de existir. Quiero que mires a tu esposo de entonces, no como a mí, el hombre que te pide esta promesa, sino sólo como a su yo posterior corrupto" (Parfit, 1984; citado por Schechtman, 1996:24). La pregunta que se plantea aquí es cómo la esposa honraría mejor los deseos del hombre con el que se casó.

Otro ejemplo hipotético que Schechtman (1996:87-88) ofrece para ilustrar la pregunta de la caracterización se refiere a una mujer que ha sido víctima de un cónyuge violento y abusivo durante mucho tiempo, lo que ha producido cambios marcados en su personalidad. Esta mujer se ha vuelto tímida y temerosa, ha roto relaciones centrales en su vida, ha reprimido sus propios deseos y se ha concentrado en hacer y ser lo que su abusador requiere. Esta mujer, además, tiene problemas

\footnotetext{
${ }^{3}$ Los datos empíricos que aquí se presentan son resultado de veintiséis entrevistas semiestructuradas a estudiantes de medicina, médicos generales y médicos especialistas de México (ocho, nueve y nueve participantes, respectivamente). Véase Villanueva (2019) para más detalles de la metodología.
} 
para identificarse con la adolescente vivaz que ve en una fotografía suya del bachillerato, a pesar de que sabe que ella es el ser humano del retrato. Le resulta imposible recordar cómo se sentía cuando la vida parecía llena de promesas, o que quería ser periodista, o en qué tipo de casa quería vivir. A veces es incapaz de conocer sus verdaderos deseos, sino sólo aquellos que su abusador desea que tenga.

Estos dos ejemplos se asemejan en que suponen cambios en la caracterización de un individuo que nos hacen cuestionarnos si, tras esos cambios, estamos frente a la misma persona. Cabe preguntarse, sin embargo, qué tipo de cambios en la caracterización valen para plantearse tal cuestionamiento, pues todas las personas cambiamos a lo largo del tiempo y en distintos contextos sociales. Lo que yo propongo es que deben ser modificaciones capaces de fracturar la experiencia de continuidad que las personas tenemos sobre nuestra propia identidad. Esto es lo yo denomino el problema de la transformación.

Desde una perspectiva psicológica, Prebble, Addis y Tippet (2013) muestran que nuestra experiencia de continuidad depende de varios factores. Por un lado, nuestras interacciones corporales con el mundo nos otorgan un sentido subjetivo de uno mismo (SSUM), prerreflexivo, con el cual nuestras experiencias adquieren un "sabor fenomenológico" de propiedad sobre nuestro cuerpo, nuestros estados mentales y nuestras acciones. Este SSUM posibilita la capacidad de autoconsciencia (identificarme a mí mismo como yo mismo) y acompaña nuestros "viajes imaginarios en el tiempo" a través de la memoria autobiográfica e historias imaginarias de uno mismo (consciencia autonoética). Todos estos elementos psicológicos se conjuntan para darnos un sentido de continuidad fenomenológica que nos hace experimentar nuestro presente, nuestros recuerdos autobiográficos y nuestros planes a futuro como propios.

Aunado a ello, nuestra memoria autobiográfica nos dota de información para construir el concepto que tenemos de nosotros mismos. Visto a través del tiempo, este concepto de uno mismo nos da un sentido de continuidad narrativa: recordamos historias que nos dicen quiénes hemos sido y seremos a lo largo del tiempo. Las narrativas sobre uno generan, a su vez, continuidad semántica, esto es, un concepto de uno mismo abstraído de esas historias que se extiende en el tiempo (Prebble, Addis y Tippet, 2013).

La continuidad narrativa y semántica descrita por estas psicólogas subyace a las teorías narrativas sobre la identidad personal. En contraste con algunas de esas teorías que suscriben que nuestra identidad es una historia en desarrollo, ${ }^{4}$ Lin-

\footnotetext{
${ }^{4}$ Por ejemplo, la teoría que ofrece Schechtman (1996) en su primer libro, The Consitution of Selves.
} 
demann (2001) sostiene que la identidad personal está formada por un tejido de historias o fragmentos de historias. Este conglomerado narrativo contiene historias que representan las diferentes facetas de las personas, incluyendo narrativas grupales - que se refieren a los grupos sociales a los que pertenecemos-y narrativas personales - que revelan cómo una persona se diferencia de los demás individuos. Las narrativas grupales y personales se combinan para formar historias retrospectivas que constituyen mi entendimiento sobre quién he sido en relación con otras personas y, al mismo tiempo, estas historias retrospectivas allanan el campo de acción en el que construyo las historias prospectivas imaginarias que guían mis acciones futuras y mi futura relación con otras personas (Lindemann, 2001). Dicho de otro modo, la identidad narrativa sirve como una lente que filtra nuestra experiencia y nuestros planes futuros. De acuerdo con esta metáfora de la lente, nuestras historias retrospectivas sirven como una lente a través de la cual experimentamos el mundo; como un molde que estructura nuestras relaciones con los objetos, con las demás personas y con nosotros mismos; como un marco interpretativo que media todas nuestras experiencias. Esto quiere decir que las narrativas sobre uno mismo organizan nuestra experiencia.

Como asenté anteriormente, yo entiendo el problema de transformación como aquel cambio en la individualidad que fractura la experiencia de continuidad de una persona. En el experimento mental de Parfit, por ejemplo, el joven ruso tiene una experiencia de discontinuidad con la historia prospectiva que hace de sí mismo, pues la diferencia de perspectiva entre él ahora (en el momento de redactar el documento para ceder las tierras) y él en el futuro posible (en que quiere revocar la concesión de las tierras) es tan significativa para él, que supone una ruptura tanto en su continuidad semántica — no se identifica con el heredero corrompido por la riqueza en el que podría convertirse (i.e. "yo no soy esa persona")—como en su sentido continuidad fenomenológica (i.e. "si pierdo estos ideales, habré dejado de existir"). En el ejemplo planteado por Schechtman, la víctima de abuso tiene también tiene una experiencia de discontinuidad, pero en este caso con una historia retrospectiva. Aquí, la diferencia de perspectiva entre ella ahora (como mujer violentada) y ella en el pasado (en la foto del bachillerato) es tan significativa que ella ahora no puede identificarse con la chica vivaz del retrato, con los sueños que ella tenía y con sus emociones de esperanza, pues lo que sabe de sí misma ahora es muy distinto a lo que sabía de ella entonces (ruptura de la continuidad semántica) de tal forma que no puede sentirse como la misma persona, aunque sabe que es ella la chica de la fotografía (ruptura de la continuidad fenomenológica).

Este par de casos hipotéticos dejan entrever que, en los casos de transformación, las personas experimentan una discontinuidad ya sea en el sentido subjetivo 
de uno mismo (discontinuidad fenomenológica), en el concepto que tienen de sí mismos (discontinuidad semántica) o en las historias que componen su identidad (discontinuidad narrativa). Los datos empíricos sobre lo que implica "transformarse en médico" ofrecen narrativas de discontinuidad reales que, como veremos enseguida, permiten entender con mayor profundidad los cambios que precipita una transformación en el conglomerado narrativo que constituye nuestras identidades personales en tanto individualidades.

\section{Transformarse en médico: narrativas de discontinuidad}

Yo estudié medicina y viví en carne propia la transformación personal que conlleva formarse como médico. Para mí, esa transformación fue ambivalente: a veces me produjo experiencias gratificantes, pero muchas veces más me condujo a experiencias insatisfactorias y dolorosas. En varios episodios fui consciente de cómo me estaba convirtiendo en una persona que no quería ser; tuve, pues, varias experiencias de discontinuidad fenomenológica, narrativa y semántica.

En mi investigación doctoral (Villanueva, 2019) quise saber si otros médicos y estudiantes habían vivido su propia transformación de manera similar a mí. En el material empírico recopilado encontré dos tipos de narrativas diferentes sobre cómo estudiar medicina transforma a los médicos en el sentido de la pregunta de la caracterización. Mientras que un grupo de narrativas describen su transformación como una "mejoría" de la identidad, el otro grupo de narrativas describe este proceso más bien como un "deterioro". En ambos casos, sus narrativas ilustran experiencias de discontinuidad que vale la pena analizar.

Proveniente de una familia indígena de Oaxaca, Guadalupe estudió medicina en una universidad pública de la Ciudad de México y, diez años después de haber egresado, tiempo durante el cual ejerció como médica general, hizo la especialidad en rehabilitación. Para ella, convertirse en médica ha sido motivo de orgullo:

[Ser médica] ha interferido muchísimo en mi forma de ser, en mi forma de comportarme. Yo era muy tímida, muy tímida, mi esposo no me cree, 5 y el hecho de ser médico pues me ha dado seguridad, confianza como para desenvolverme en algunos lugares. Igual me pone como en una situación de autoridad, no sé si intelectual o moral, en mi familia. Nadie ha hecho una carrera universitaria, sólo yo [...] Entonces sí, por supuesto que [ser médica] ha influido, me ha dado cierta confianza, muchísimo más a partir de la especialidad. (Guadalupe, rehabilitóloga, 42 ańos).

\footnotetext{
5 Para este y los demás casos, los énfasis en cursiva son de la autora del artículo.
} 
En esta cita podemos apreciar la confrontación que hay entre la caracterización retrospectiva que Guadalupe hace de sí misma- "yo era muy tímida, muy tímida" - con la caracterización actual que hace de sí-"ser médico pues me ha dado seguridad, confianza como para desenvolverme". Estas caracterizaciones sobre sí son tan contrastantes que Guadalupe incluso expresa que su esposo no le cree que ella antes era de esa otra manera, pues esa caracterización retrospectiva no concuerda con las historias vigentes de su conglomerado narrativo. Este ejemplo ilustra la discontinuidad semántica que hay entre la Guadalupe tímida del pasado y la Guadalupe segura del presente.

Para otros entrevistados, transformarse en médico los ha llevado a ser una persona que no querían ser y que no reconocen, como muestran las siguientes dos citas:

Yo recuerdo en algún momento que me convertí en el monstruo residente, ${ }^{6}$ fue en Medicina Interna, por tanto paciente, tanto trabajo que era. [...] Y había un paciente moribundo y realmente llegué a pensar: "Espero que ya se muera para que no tenga ya más trabajo porque hoy estoy atoradísimo". Y no, el paciente no falleció, pero llegar a ese lugar sí fue para mí, pues fue un momento en el que sí me tuve que parar y decir: "a ver, ¿qué te pasa?, tú no eras alguien asi". (Ramón, estudiante, 28 años).

Llegó un momento en que yo llegaba todos los días enojada [a la clínica], y de hecho sí llegué a ser muy, muy dura con algunos pacientes, así de que "si quiere, y si no, no lo haga". Así de: "el que sigue". Sí llegó un momento en el que me empecé a volver así. Y fue cuando agarré y dije: "¿Qué me está pasando? Esta no soy yo". O sea, no era la [misma] persona a la que [años atrás] en el hospital las enfermeras adoraban. (Jazmín, médica general, 43 años).

Las experiencias de discontinuidad de Ramón y Jazmín son muy claras: no se identifican con la persona que están siendo, y por eso se cuestionan qué está pasando con ellos pues sienten que "esta persona no soy yo". Estos casos ilustran experiencias de discontinuidad fenomenológica en donde está interrumpido el sentido subjetivo de uno mismo, lo que impide que Ramón y Jazmín sientan como propias sus acciones.

Además, en la cita de Jazmín podemos apreciar un conflicto narrativo que es producto del choque que se da entre las historias que conforman el conglomerado narrativo de una persona y las nuevas historias que se integran a éste como resultado de la transformación. En este ejemplo chocan las nuevas historias que se están integrando al conglomerado narrativo de Jazmín ("llegaba enojada a la clínica y era muy dura con los pacientes") y sus historias retrospectivas de las que ella abstraía

\footnotetext{
${ }^{6}$ En esta cita y otras posteriores, al hablar de "residentes" se hace referencia a los médicos que están realizando prácticas en un hospital con el fin de formarse como especialistas.
} 
un concepto ella misma muy diferente al actual ("en el hospital las enfermeras me adoraban").

Lindemann (2001) defiende que las historias que conforman nuestros conglomerados narrativos conllevan una serie de compromisos con otras personas, con ideas, causas, valores, proyectos, etc. Como muestran los ejemplos anteriores sobre experiencias de discontinuidad, a veces, el conflicto que revelan las nuevas historias que se integran al conglomerado narrativo a partir de la transformación personal implica cambios en estos compromisos, los cuales también forman parte del concepto de uno mismo y de las historias prospectivas y planes a futuro. Esto se aprecia también en esta otra cita:

Cuando hablas con los residentes es muy común que empiecen diciendo "No, pues yo quería hacer algo interesante de la Medicina, hacer lo que me gusta", y poco a poco van pensando en dinero y trabajar menos [...] Cosas así, que vas cambiando tu forma de vida de inicialmente piensas en el paciente, en lo que te parece interesante, y al final lo único que quieres es ya, hacer lo menos posible, y hacer dinero [...] Es un proceso así, lo que quieres es simplemente irte y ganar dinero y ya hacer lo menos posible, porque en realidad, por alguna razón, les deja de gustar lo que hacen. (Rodolfo, estudiante, 23 años).

En suma, como hemos visto hasta ahora, las transformaciones personales conllevan un conflicto narrativo que puede manifestarse como un conflicto en el concepto de uno mismo (ejemplo de Ramón, Jazmín y de la mujer violentada de Schechtman), en las expectativas que los demás tienen sobre uno con base en nuestro conglomerado narrativo (ejemplo de Guadalupe) o en los compromisos pasados y futuros (ejemplo de Rodolfo y del joven ruso de Parfit).

Sin embargo, en los casos de transformación personal muchas veces no sólo cambia el contenido de la identidad narrativa, sino que la integración de nuevas historias también modifica la propia organización del conglomerado narrativo, es decir, el orden de importancia que tienen las diferentes historias para conformar la identidad de la persona. Lindemann (2001) reconoce este orden en la conformación de la identidad al hablar del peso (heft) que tienen las historias que componen nuestro conglomerado narrativo. Para ella, el peso de una historia debe ser proporcional a cuánto importa lo que representa esa historia para definir quién soy. En el ejemplo anterior de Jazmín vemos que para ella era muy importante la historia que la representaba como alguien adorable, pero después de su transformación esa historia había perdido peso, pues ahora tenía historias que la representaban más bien con un carácter iracundo. Dicho de otro modo, su transformación había reorganizado el peso de las historias que componen su conglomerado narrativo. 
Otras disciplinas también han planteado la idea de que nuestra identidad tiene un orden que organiza quiénes somos. Desde la perspectiva de los estudios de género, por ejemplo, Serret (2011) defiende que nuestras identidades sociales tienen un arreglo jerárquico, siendo el género la de mayor jerarquía que organiza el resto de nuestra identidad. Desde la perspectiva psicoterapéutica, los roles sociales representan diferentes "voces" de la persona. Las voces más dominantes narran la mayor parte de las historias autobiográficas, aplicando su punto de vista y sus "reglas generales". En términos psicoterapéuticos, una transformación de la identidad ocurre cuando cambia la voz dominante y/o el contenido de las narrativas autobiográficas (Ribeiro y Gonçalves, 2010).

Transformarse en médico implica una reorganización de este tipo en el conglomerado narrativo de los estudiantes de medicina. Para Braulio, uno de los estudiantes entrevistados, la identidad médica es tan poderosa para estos profesionales que en entrevista comentó:

Mientras vas estudiando medicina también te vas haciendo médico, o sea como que tu personalidad ya no eres tú, eres médico [...] ya no soy Braulio, soy Braulio el médico, ¿̇me explico? Una de sus características, o no ni siquiera una, todo él es un médico antes de ser él mismo. Yo creo que es claro, antes de ser persona, son médicos. (Braulio, estudiante, 26 años).

Esta reorganización de la identidad también se ve reflejada en un texto que yo misma redacté durante el último año de la carrera de medicina, mientras hacía el servicio social en un pequeño poblado del sureste mexicano:

[...] llegó un adolescente tocando mi puerta. Guillermina, su madre analfabeta, tenía un dolor abdominal que la retorcía en la cama. Cuando llegué a su humilde vivienda, interrogué a Guillermina con paciencia porque ya la conozco y bien sé que siempre responde a algo diferente a lo que le pregunto. [...] Muchas veces mis palabras técnicas me distancian de mis pacientes [...] Al ser médico se espera que uno deje de ser Juan o Luis para convertirse en el doctor García o Sánchez, y asi todo lo conocido sufre una metamorfosis solemne: la "caca" se convierte en "heces", las "agruras" en "pirosis", el "pecho" en "tórax", las flemas en "esputo" [...] Si alguna vez me salió de un yo antiguo decir "me duele la garganta" frente a alguno de [mis profesores], siguió una reprimenda con el fin de avergonzarme en público. “ $¡ N o$ hable como si fuera verdulera! Usted es médico. No se dice 'dolor de garganta', se dice 'odinofagia”. Por eso, incluso yo que luché contra todos esos protocolos inanes y acorté distancias entre el consultor y el consultante, le pregunté a Guillermina:

- ¿Y ha podido arrojar gases?

— ¿Pedos? - preguntó con cara de incomprensión.

— Sí. ¿Ha podido echarse peditos? — ¡minimicé estúpidamente la palabra!, como si me incomodara.

— Sí. Pedos sí tengo. Es que aquí les llamamos así: "pedos".

Y pensé con nostalgia: “yo también les llamaba 'pedos'...” (Villanueva, 2011:74). 
En esta cita, el cambio en la organización de jerarquías en la identidad se manifiesta a través de la permuta del nombre de pila de la persona por el apelativo "doctor", lo que no es un cambio inocuo. ${ }^{7}$ Además, la metamorfosis de la terminología empleada para describir cosas ordinarias puede interpretarse como un cambio en la voz dominante que narra nuestras historias. Este cambio de "voces" del que se habla en el contexto psicoterapéutico es un cambio de narrador, producto de la reorganización de la jerarquía de las historias que componen nuestro conglomerado narrativo tras una transformación personal. Cuando se completa la transformación, la voz de esta "nueva" identidad se vuelve el narrador dominante de las historias autobiográficas que componen nuestro conglomerado narrativo, otorgándoles un sentido distinto. De ahí que en este escrito me refiera a un "yo antiguo" y que sienta nostalgia por él.

Es importante tener en cuenta que con la reorganización de nuestro conglomerado narrativo no sólo cambia el narrador de nuestras historias retrospectivas y prospectivas, sino que sobre todo se transforma nuestra fenomenología. Recordemos que como expliqué anteriormente con la metáfora de la lente, las narrativas sobre uno mismo organizan nuestra experiencia, es decir, nuestro conglomerado narrativo funciona como una lente que filtra nuestra experiencia y nuestros planes futuros. Este cambio de fenomenología puede apreciarse claramente en el siguiente relato:

En una de mis primeras guardias [...] teníamos que poner un catéter, haces un hoyito abajo del ombligo, y es una varilla como de este tamańo [...] para diálisis, metes el catéter y oyes cuando truena, y pues la residente tampoco era muy hábil que digamos, pues acabó metiendo la varilla y perforó el intestino. Cuando vimos, las zanahorias ya estaban en el catéter. Lo puso a final de cuentas la residente, pero como yo estaba con ella, yo dije "Es que ya la matamos". [...] Había pacientes alrededor y la [residente] me dice: “A ver, ven, ¿`cómo se te ocurre? Eso no se dice”. Ay no, bueno, viene la [médica del turno] de la noche e igual: "Es que eso no se dice, eres esto, eres aquello, no te quiero en mi servicio". Ya le hablaron al cirujano, la operaron, pero pues la paciente falleció [...] Esa guardia sí me la pasé muy mal, porque me regańaron por todos lados, pero yo en ese momento a lo mejor no tenía la experiencia o la prudencia, sino que me espanté [...] Ya después con más calma la [residente] me dijo: "Es que de diez catéteres que vas a poner, a la mejor nueve te van a quedar bien, y uno te va a salir mal. Éste fue el que salió mal. No la matamos, fue esto, fue esto, fue esto. ¿Cómo vas a aprender, si no lo haces?” Me quedó mucho lo que decía, "el único que no se equivoca es el que no lo hace". (Natalia, médica internista, 37 años).

\footnotetext{
Como asienta Goffman, un renombrado sociólogo que estudió la dimensión social de las identidades, "Siempre que una ocupación lleve implícito un cambio de nombre, registrado o no, podemos estar seguros de que existe una importante fractura entre el individuo y su mundo anterior" (Goffman, 2006[1963]:75).
} 
Analicemos con detenimiento la transformación en la fenomenología de Natalia. Para empezar, Natalia-estudiante, en una de sus primeras guardias, filtró esta experiencia con su conglomerado narrativo pretransformación, y la vivió como un caso claro de iatrogenia en el que una paciente falleció como consecuencia de las acciones de una residente. La narradora de esa experiencia (Natalia-estudiante) la describió así: "es que ya la matamos". Esta experiencia es muy diferente de las experiencias que ha tenido después Natalia-especialista, como podemos ver en el resto de su relato:

[Con el tiempo,] pues vas agarrando también la prudencia de qué debes y qué no debes decir. $Y$ sobre todo te vas haciendo, te vas como curtiendo, la verdad, y vas viendo quién sale y quién no sale. $\mathrm{O}$ sea, no vas a sacar bien a todos, los vas viendo y también te vas mentalizando de que pues se van a morir [...] Sí te das cuenta de que hay cosas que a la mejor te equivocas, porque infalible no eres. Te equivocas y lo vas diciendo: "Sí, me equivoqué, pero aprendí. Con el siguiente no me vuelve a pasar". Te dejas de sentir, al principio te sientes muy culpable de decir se me murió, y ya no dices se murió, dices "me equivoqué", o no sé, "a la mejor si hubiera hecho esto hubiera aguantado más" [...] Pero con el tiempo te vas dando cuenta que dices: "bueno, se iba a morir de cualquier manera, sí se iba a morir" [...] Te das cuenta de que no te equivocas, que no lo haces con dolo, sino dices "bueno, yo en ese momento pensé que era lo más adecuado para ese paciente, me equivoqué". (Natalia, médica internista, 37 años).

En este último fragmento del relato de Natalia podemos apreciar cómo su conglomerado narrativo ha sido reorganizado por el proceso de transformación ("te vas como curtiendo"), por lo que Natalia-especialista filtra de manera muy distinta sus experiencias a como lo hacía Natalia-estudiante. La narradora post-transformación relata: "ya no dices se me murió, dices me equivoquê". Mientras que en la historia que vivió y narró Natalia-estudiante las acciones de la residente se presentan como causas claras del fallecimiento de la paciente y está impregnada de sentimientos de culpabilidad, la historia que narra Natalia-especialista tiene un tono más justificatorio, la relación entre causas y efectos es menos evidente ("se iba a morir de cualquier manera"), y los sentimientos de culpabilidad están atenuados. Es en este sentido que la reorganización del conglomerado narrativo que ocurre con una transformación personal no sólo modifica el contenido de la identidad, sino también su arreglo jerárquico y, por ende, la fenomenología de la persona transformada.

\section{Reparar la discontinuidad: los argumentos autobiográficos}

La identidad personal es resistente al cambio. En términos psicoanalíticos, Ribeiro y Gonçalves (2010) han señalado que, ante cambios con potencial transformativo que producen ambivalencia y conflicto emocional, las personas tienden a atenuar 
los cambios, minimizándolos, despreciándolos o trivializándolos para conservar su individualidad. Ante la inestabilidad, explican estos psicoanalistas, las personas preferimos lo conocido, lo seguro, lo familiar, y rechazamos lo desconocido, lo inseguro, lo extraño. Ese rechazo a lo desconocido suele estar mediado por emociones negativas como el miedo y la aversión. Estos mecanismos psicológicos buscan conservar la organización jerárquica de nuestro conglomerado narrativo y mantener así la voz narrativa que ha sido dominante hasta ese momento, es decir, buscan prevenir la transformación.

Ricœur explica la resistencia que tenemos al cambio al plantear que las personas tenemos el compromiso ético de preservarnos a lo largo del tiempo: "aunque cambie mi deseo, aunque yo cambie de opinión, de inclinación, 'me mantendré" (Ricœur, 1996:119). Para el filósofo francés, esta promesa de mantenerme surge de la obligación moral de hacerme responsable ante la confianza que los otros depositan en mí. Si me prestas dinero, esperarás que yo me mantenga a mí misma como tu deudora que prometió pagarte en tiempo, por ejemplo. La promesa de mantenerse de Ricoeur es necesaria para las interacciones sociales en un sentido muy fundamental, pues justifica y posibilita la confianza en el otro. Cuando esta promesa se rompe, defraudamos la confianza y las expectativas que los otros tenían sobre nosotros y podemos perder relaciones importantes. Por eso, las transformaciones personales no son frecuentes: sus costos sociales y emocionales son muy altos.

Ante experiencias de discontinuidad las personas tendemos a idear narrativas que nos permitan integrar las nuevas historias a nuestro conglomerado narrativo. Esto busca prevenir o en su defecto reparar la discontinuidad fenomenológica, narrativa y semántica ("esta no soy yo") que caracteriza al conflicto narrativo que conlleva la transformación personal. A veces, el trabajo narrativo de integración consiste en reinterpretar nuestras historias retrospectivas de tal forma que las nuevas historias parezcan seguirlas naturalmente- "como historiadores whiggistas" ${ }^{8}$ diría el filósofo Raymond Martin (1998:117-118), quien plantea cuestiones muy interesantes para la individualidad. ${ }^{9}$ Esto se consigue mediante la apropiación de las nuevas historias

\footnotetext{
${ }^{8}$ El término "whig history" fue acuñado por Butterfield en 1931 y conlleva una connotación crítica hacia un tipo de historiografía en el que la historia se presenta a través de grandes narrativas presentistas, es decir, como un progreso de un pasado oscuro a un presente glorioso (para una discusión más profunda, véase Wilson y Ashplant, 1988).

9 Martin (1998) trabaja el problema de la transformación personal como una sustitución de yoes que se da a través de la apropiación de un yo sustituto (a surrogate self). Para él, una persona se transforma cuando se identifica con otro yo, el cual sustituirá a su yo actual a través de un proceso que implica empatía (ponerte emocional o cognitivamente en el lugar del otro yo), simpatía (ser empático y además adoptar objetivos del otro yo) y apropiación. Esta última está constituida por tres elementos: 1) afectarte de una manera que sólo puede ser experimentada por alguien que recuerda o imagina experiencias propias; 2) contextualizar cognitivamente las experiencias apropiadas "como historiadores whiggistas"; y 3) comportarte como si las experiencias del otro yo fueran propias.
} 
y el abandono de algunas historias retrospectivas. ${ }^{10}$ En sus estudios sobre la memoria autobiográfica, Conway, Singer y Tahini (2004) señalan que el "yo recordado" es distorsionado sistemáticamente de manera automática para ajustarlo al concepto de uno mismo que tenemos en el presente, con el propósito de asegurar la continuidad semántica de uno mismo a lo largo del tiempo.

Cuando las personas no conseguimos reparar la discontinuidad de esa manera, creamos argumentos autobiográficos para recobrar la sensación de continuidad. Habermas y Köber (2015) describieron dos tipos de argumentos autobiográficos: el argumento esencialista, según el cual las personas se convencen de tener una esencia (es decir, una individualidad) que perdura a través de su transformación, y el argumento narrativo, que cuenta la historia de cómo ha ido cambiando la persona, recobrando con el hilo narrativo la sensación de continuidad. La importancia de estos argumentos no recae en su veracidad, sino en su capacidad para prevenir y reparar la discontinuidad fenomenológica, narrativa y semántica que caracteriza el conflicto emocional de las transformaciones personales.

Muchas de las citas utilizadas en este artículo para ilustrar el proceso de transformación de los médicos son precisamente argumentos narrativos en los que los entrevistados cuentan la historia de cómo han ido cambiando. Los médicos que ofrecieron argumentos esencialistas son los de mayor edad entre mis entrevistados, y con una muy buena posición jerárquica dentro del campo médico. Esto parece apuntar que los argumentos esencialistas son más comunes entre médicos que completaron su transformación hace muchos años. Aquí un ejemplo:

Marcia: ¿Cambió su forma de ser de alguna manera al estudiar medicina?

Renato: Sí, no sabría decirlo. Siempre fui de carácter fuerte, fuerte, pero en el buen sentido de la palabra, no aquel machista ni aquel que grita, me refiero yo a soportar los golpes, a soportar los castigos, a soportar las adversidades. Siempre tuve un carácter fuerte, pero a la vez siempre he sido muy humilde, muy humilde en aceptar errores, en aceptar lo que puedo y lo que no puedo hacer, entonces eso, yo creo que eso me ayudó. (Renato, neurocirujano, 43 años).

\section{Conclusiones}

En este artículo definí el problema de la transformación como una variante del problema de la individualidad referente a la persistencia de la identidad personal, enfo-

\footnotetext{
${ }^{10}$ Lindemann (2014) defiende que nuestros conglomerados narrativos van cambiando a lo largo del tiempo en la medida en que sujetamos (holding) ciertas historias mientras que dejamos ir (letting go) otras.
} 
cada en la pregunta de la caracterización. Argumenté que dicho problema se refiere a cambios profundos en la caracterización de una persona capaces de producir una experiencia de discontinuidad ya sea en el sentido subjetivo de uno mismo (discontinuidad fenomenológica), en el concepto que tienen de sí mismos (discontinuidad semántica) o en las historias que componen su identidad (discontinuidad narrativa).

Desafiando la metodología tradicional de la filosofía de la identidad personal basada en experimentos mentales, utilicé datos empíricos con narrativas de discontinuidad reales sobre lo que implica "transformarse en médico" para explorar el problema de la transformación y las experiencias de discontinuidad que conlleva. Esto permitió identificar que dicho problema se acompaña de un conflicto narrativo que es producto del choque que se da entre las historias que conforman el conglomerado narrativo de esa persona y las nuevas historias que se integran a éste. El análisis de los datos empíricos mostró que el conflicto narrativo muchas veces también modifica la propia organización del conglomerado narrativo, lo que a su vez transforma la fenomenología de la persona.

Por último, señalé que las transformaciones personales no son tan comunes como parecen, ya que nuestras identidades son resistentes al cambio para preservar la individualidad. Ante situaciones con un potencial transformador, las personas minimizamos los cambios o bien reinterpretamos nuestras historias retrospectivas de tal forma que las nuevas historias parezcan seguirlas naturalmente. Pero cuando esto no funciona y el conflicto narrativo persiste, creamos argumentos autobiográficos para recobrar la sensación de continuidad, ya sea convenciéndonos de tener una esencia que sobrevivió a los cambios (argumento esencialista), o bien, contando la historia de nuestros cambios (argumento narrativo). 


\section{REFERENCIAS BibLIOGRÁFICAS}

Conway, M., Singer, J., y Tahini, A. (2004). The Self and Autobiographical Memory: Correspondence and Coherence. Social Cognition, 22(5), pp. 491-529. DOI: https://doi.org/10.1521/soco.22.5.491.50768

Dennett, D. (1978) Where am I? In Brainstorms. MIT Press.

Goffman, E. (2006[1963]). Estigma. La identidad deteriorada. Amorrortu.

Habermas, T., y Köber, C. (2015). Autobiographical reasoning in life narratives buffers the effect of biographical disruptions on the sense of self-continuity. Memory, 23(5), 664-674. DOI: https://doi.org/10.1080/09658211.2014.920885

Lindemann, H. (2001). Damaged identities, narrative repair. Cornell University Press.

Lindemann, H. (2014). Holding and Letting Go: The Social Practice of Personal Identities. Oxford Scholarship Online. DOI: https://doi.org/10.1093/acprof:oso/9780199754922.001.0001

Locke, J. (1982[1690]). Ensayo sobre el entendimiento humano. Fondo de Cultura Económica.

Martin, R. (1998). Self-concern: an experiential approach to what matters in survival. Cambridge University Press. DOI: https://doi.org/10.1017/ CBO9780511663826

Muñoz Corcuera, A. (2021). Persistence Narrativism and the Determinacy of Personal Identity. Philosophia, 49 (2): 723-739. DOI: https://doi.org/10.1007/ s11406-020-00265-8

Olson. E. (2010). Personal Identity. Stanford Encyclopedia of Philosophy, Winter 2010 Ed. Stanford University. (https://plato.stanford.edu/archives/win2010/entries/identity-personal/).

Parfit, D. (1984) Reasons and Persons. Clarendon Press.

Prebble, S., Addis, D. y Tippet L. (2013) Autobiographical Memory and Sense of Self. Psychological Bulletin, 139(4), 815-840. DOI: https://doi.org/10.1037/ a0030146

Ribeiro, A., y Gonçalves, M. (2010). Commentary: Innovation and Stability within the Dialogical Self: The Centrality of Ambivalence. Culture y Psychology, 16(1), 116-126. DOI: https://doi.org/10.1177/1354067X09353211

Ricœur, P. (1996). Si mismo como otro. Siglo XXI. 
Serret, E. (2011). Hacia una redefinición de las identidades de género. Géneros, (9), 71-97.

Schechtman, M. (1996). The constitution of selves. Cornell University Press.

Villanueva, M. (2011). Cacaos. Seminario de Medicina y Salud, Facultad de Medicina, UNAM.

Villanueva, M. (2019). Somos médicos, no dioses: una etnografía filosófica de la deshumanización de la medicina (Doctorado en Filosofía de la Ciencia). UNAM. (Disponible en: https://tesiunam.dgb.unam.mx/F/RD6VVYMT98365E7XLMF4RYMN17UJYLFJVUY14DNI2SBUJNYXYE-07915?func=full-set-set\&set_number $=824442 \&$ set_entry=000001\&format=999)

Wilkes,K.(1993).RealPeople:PersonalIdentitywithout ThoughtExperiments. Oxford UP. DOI: https://doi.org/10.1093/acprof:oso/9780198240808.001.0001

Wilson, A., y Ashplant, T. (1988). Whig history and present-centred history. The Historical Journal, 31(1), 1-16. DOI: https://doi.org/10.1017/ S0018246X00011961

DOI: https://doi.org/10.15366/bp2021.28.009

Bajo Palabra. II Época. No28. Pgs: 199-216 
